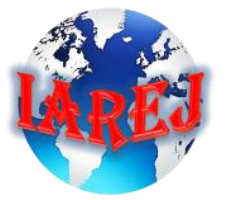

Research Article

\title{
Synthesis and stability analysis of folic acid-graphene oxide nanoparticles for drug delivery and targeted cancer therapies
}

\author{
Neşe Keklikcioğlu Çakmak ${ }^{a * *}$ (D), Mustafa Küçükyazıcı ${ }^{a}$ (D) and Atakan Eroğlu ${ }^{a}$ \\ ${ }^{a}$ Department of Chemical Engineering, Cumhuriyet University, Sivas 58140, Turkey
}

\begin{tabular}{l}
\hline ARTICLE INFO \\
\hline Article history: \\
Received 02 April 2018 \\
Revised 01 September 2018 \\
Accepted 10 September 2018 \\
\hline Keywords: \\
Cancer \\
Nano-graphene oxide \\
Folic acid \\
Nano-drug delivery systems \\
Zeta potential
\end{tabular}
\begin{abstract}
Cancer is the growth and proliferation of damage-ending cells in an uncontrolled or abnormal way. Today, it takes place among the most important health problems around the world and in our country. Surgery, radiotherapy, and chemotherapy are the main treatment methods in cancer treatment. The development of resistance to chemotherapeutic medicines has led scientists to investigate this issue as well as the drug's ability to reach the targeted tumor site and destroying cancer cells in addition to normal cells. The production of various nanostructures for anticancer drug development has been one of the most important areas of nanomedicine. Thus, in the present research, the improved Hummers' method was employed for the synthesis of graphene oxide nanoparticle (NGO), and it was activated by the folic acid (FA) antibody to increase targeting ability after attachment of the drug to the nanostructure systems. SEM, FTIR, XRD, UV/Vis spectroscopy, and zeta potential analysis were performed for characterization of the products. The highest absorbance of the FA-NGO/DIW nanostructures produced at the concentration of 0.01 $\mathrm{mg} / \mathrm{ml}-0.05 \mathrm{mg} / \mathrm{ml}$ synthesized by the Hummers' method and in the UV/Vis spectra, peaks at 232 $\mathrm{nm}$ and $270 \mathrm{~nm}$ corresponds to NGO-DIW and FA-NGO/DIW, respectively. The zeta potential value above $35 \mathrm{mV}$ was obtained in all measurements, and the NGO-DIW and NGO-FA-DIW samples maintained stability for days. These findings are consistent with the few studies in the literature, and this study will guide future studies in which nanoparticle systems will be directed to the target by binding chemotherapeutic drugs.
\end{abstract}

(C) 2019, Advanced Researches and Engineering Journal (IAREJ) and the Author(s).

\section{Introduction}

Nanoparticles (NPS) represent particles between 1 and 100 nanometers in size. In nanotechnology, nanoparticles are described as small objects acting as a whole unit with regard to their features and transport $[1,2]$. Graphenebased nanoparticles, because of their exceptional physicochemical characteristics, various surface functionalization, good biocompatibility area, and ultrahigh surface, have drawn great attention in biomedical applications, including drug delivery, biosensors, theranostics, bioimaging, etc. Two-dimensional nanomaterial - graphene was revealed for the first time in 2004 [3,4]. Graphene, which consists of a single-atomthick sheet of $\mathrm{sp}^{2}$ bonded carbon atoms that are hexagonally arrayed, presents various applications from composite materials to quantum dots [5-10]. Graphene is present in different forms, including graphene oxide (GO), graphene sheets, and reduced graphene oxide (rGO) [1113]. Its biodegradability and biocompatibility are the features that make GO be preferred in the area of biomedicine, particularly theranostics [14-15]. A lot of endeavors have been recently made to the nanosized materials' abilities with controlled drug delivery properties because they enable targeted delivery, efficient loading and the release of drugs in a controlled manner, and thus may be used successfully in biomedical practices.

The research conducted recently has shown that the use of low molecular weight targeting agents including folic acid as surface coatings is very promising for the specific cancer cell recognition and enhancement in the intracellular

* Corresponding author. Tel.: +90 3462191010 (Ext:2232)

E-mail addresses: nkeklikcioglu@ cumhuriyet.edu.tr (N. Keklikcioğlu), mkckyzc@gmail.com (M. Küçükyazıcı), ataknovv.erogluu@gmail.com (A.Eroğlu) ORCID: 0000-0002-8634-9232 (N. Keklikcioğlu Çakmak), 0000-0002-4030-9147 (M. Küçükyazıcı), 0000-0003-4544-5225 (A. Eroğlu)

DOI: $10.35860 /$ iarej. 411717 
uptake of nanoparticles [16]. FA has short chains and a small size and thus will facilitate the internalization of nanoparticles. For example, Deb and Vimala [17] synthesized a graphene-oxide-polyethylene glycol-folic acid-camptothecin (GO-PEG-FA-CPT) drug delivery system, and this system was investigated by MTT assay by utilizing $\mathrm{MCF}-7$ breast cancer cell lines. Improved anticancer activity was exhibited by the conjugate, and therefore, it might be utilized as a possible candidate for drug delivery. Folic acid-conjugated GO loaded Ce6 was determined to be a powerful candidate for active drug delivery in photodynamic therapy (PDT) [18]. The nonimmunogenic, non-toxic, and stable features of folic acid turn it into an appropriate candidate for conjugation with nanocomposites [19]. Folic acid that is conjugated with nanographene oxide and polyvinylpyrrolidone (PVP) has been indicated as an advantageous nanocomposite for chemo-photothermal therapy [20].

In this research, the improved Hummers' method was employed for the synthesis of graphene oxide nanoparticle (NGO), which was activated by the folic acid antibody to increase the targeting ability after attachment of the drug to the nanostructure systems. In our aim, firstly, nanographene oxide was synthesized and then activated by the folic acid antibody, because of which sulfonic acid groups were embedded to NGO, bringing it to a stable state under physiological conditions.

\section{Material and Method}

\subsection{Synthesis of $\mathrm{NGO}$}

The improved Hummers' method with minor modification was employed for the synthesis of GO [2124]. For the purpose of acquiring NGO, GO was broken by the ultrasonic probe at $750 \mathrm{~W}$ for a period of $100 \mathrm{~min}$. SEM, FTIR, XRD, UV/Vis spectroscopy, and zeta potential analysis were performed for characterization of the products.

\subsection{Conjugation of $F A$ with $N G O$}

FA-NGO was prepared in a manner described by Zhang et al. [23]. UV/Vis spectroscopy and zeta potential analysis were performed for characterization of FA-NGO.

\subsection{Characterization}

A scanning electron microscope (TESCAN MIRA3 $\mathrm{XMU}$ ) was used to measure the morphologies of NGO. The FT-IR spectra of NGO were characterized by an FTIR spectrophotometer (Bruker: Tensor II) in the 400-4000 cm-1 range. A diffractometer (Rigaku DMAX IIIC) was used for the purpose of obtaining X-ray diffraction (XRD) data. A UV-Vis spectrophotometer (UV-1280, Shimadzu, Japan) was used for recording the spectra of the prepared specimens in the $200-800 \mathrm{~nm}$ range. A Malvern Zetasizer Nano $Z$ was utilized for the measurement of the specimens' zeta potentials. In the end, for the purpose of the homogeneous dispersion of NGO particles in DIW, a probe sonicator (Sonics \& amp; materials INC, USA) with $750 \mathrm{~W}$ power was utilized.

\section{Results and Discussion}

An image of scanning electron microscopy (SEM) (Figure 1) provides morphological information on NGO. NGO has the appearance of black powder, is 3.1-6.8 nm in thickness, has purity $>99 \mathrm{wt} \%$ and lateral dimension of 15-40 $\mu \mathrm{m}$. As a result of technical analysis, it was demonstrated that $\mathrm{NGO}$ has the following metal contents: C-62.39\%; O-36.11\%; S-1.18\%; H- $0.32 \%$.

FT-IR tests showed the presence of $\mathrm{OH}\left(\sim 3300 \mathrm{~cm}^{-1}\right)$, $\mathrm{C}=\mathrm{C}\left(\sim 1634 \mathrm{~cm}^{-1}\right)$ [25] functional groups in NGO (Figure. $2)$.

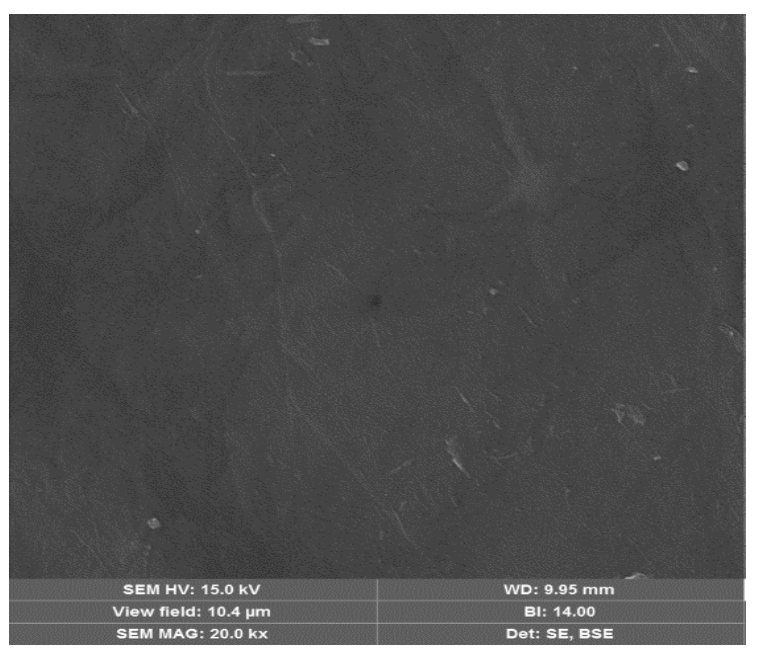

Figure 1. SEM image of NGO

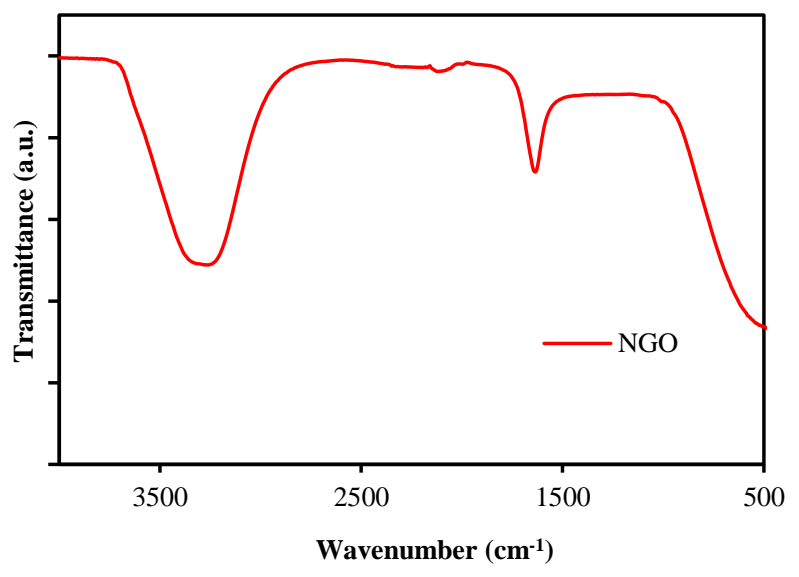

Figure 2. FT-IR spectra of NGO

Figure. 3 presents the X-ray diffraction patterns of graphite and nano-graphene oxide. Graphite shows a very strong and sharp peak at $2 \theta=26.40^{\circ}$, corresponding to the diffraction of the (002) plane. Following graphite oxidation to GO, the (002) reflection of graphite vanishes and a diffraction peak at $2 \theta=10.21^{\circ}$ exists, which 
corresponds to the diffraction of the (001) plane, indicating the oxidation of graphite in a successful way [26,27].

Figure. 4 shows the UV-Vis spectra of nano-graphene oxide. The optical absorption peak at $232 \mathrm{~nm}$, which originated from the $\pi$-plasmon of carbon [28], stayed without changes to a significant extent. The absorption peaks in the spectra at $232 \mathrm{~nm}$ and a small shoulder at 300 nm correspond to the $\pi-\pi *$ transition of aromatic $\mathrm{C}-\mathrm{C}$ bonds and the $n-\pi *$ transition of $\mathrm{C}=\mathrm{O}$ bonds, respectively.

As is seen from the UV/Vis spectra (Figure 5), a peak at $232 \mathrm{~nm}$ vanishes, whereas a new peak at $270 \mathrm{~nm}$ emerges because of the existence of FA in NGO.

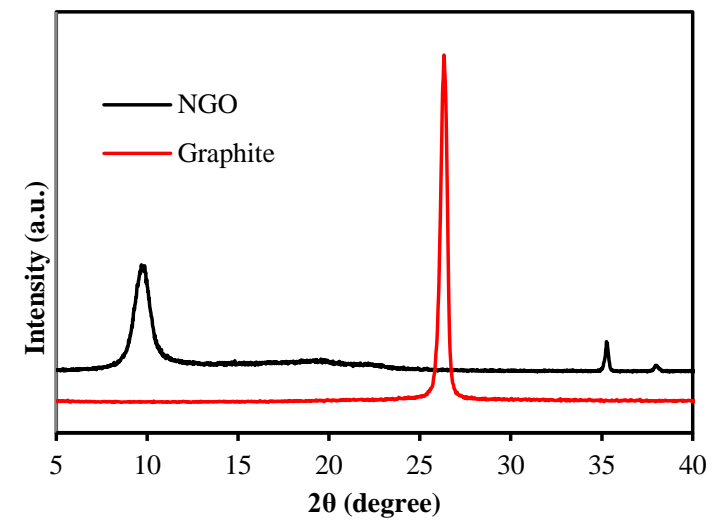

Figure 3. XRD patterns of NGO and graphite

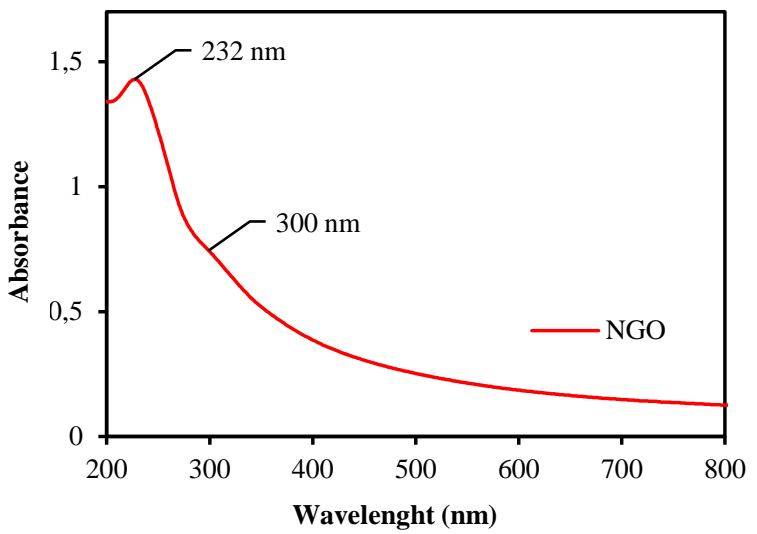

Figure 4. UV-Vis absorption spectra of NGO in DIW

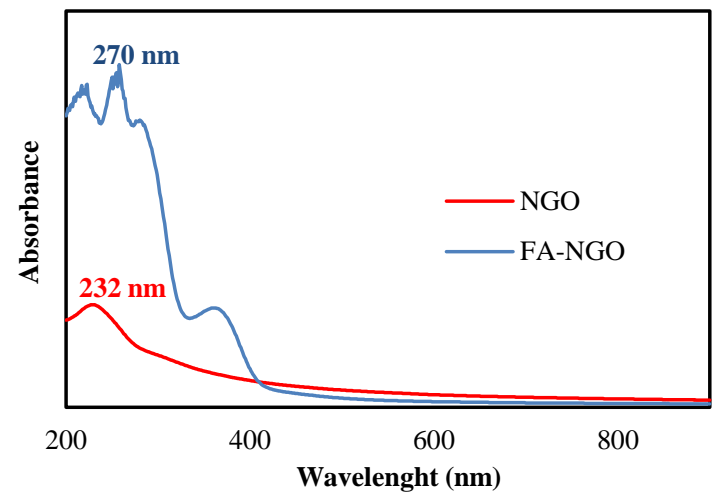

Figure 5. UV-Vis absorption spectra of NGO and FA-NGO in DIW
The stability of NGO-DIW was determined as a result of the measurement of its zeta potential values. Zeta potential represents the electrical potential between the nanoparticle surface and the base fluid, and there is a relationship between the zeta potential absolute value and nanoparticle stability. In case the measured zeta potential absolute value is above $25 \mathrm{mV}$, it is possible to say that the nanofluid produced is stable. If all the particles in suspension have a large negative or positive zeta potential, there will be a tendency for them to repel each other, but they will not tend to aggregate. The zeta potential value above $35 \mathrm{mV}$ in all measurements is shown in Figure. 6 and Figure. 7, and at the same concentration, the zeta potential value of FA-NGO is quite high compared to NGO. Therefore, dispersibility of NGO in water has been improved by the bonding of FA on NGO.

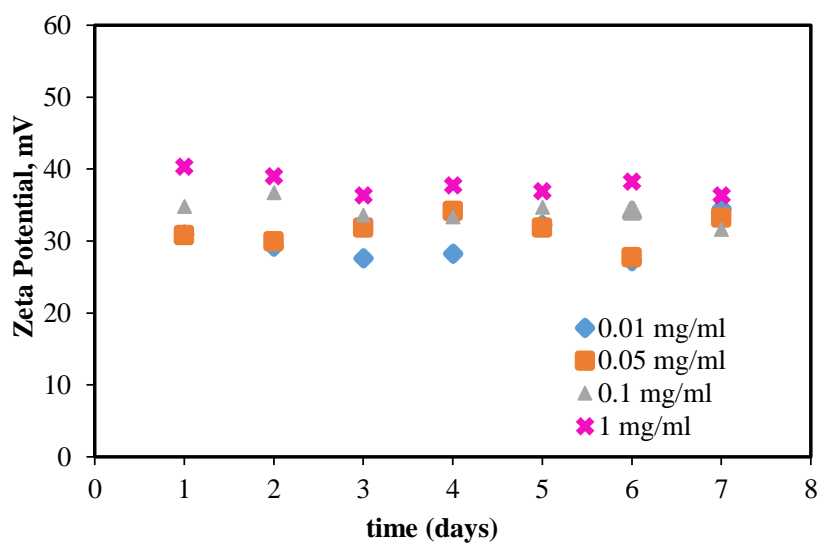

Figure 6. Stability of NGO in aqueous solutions

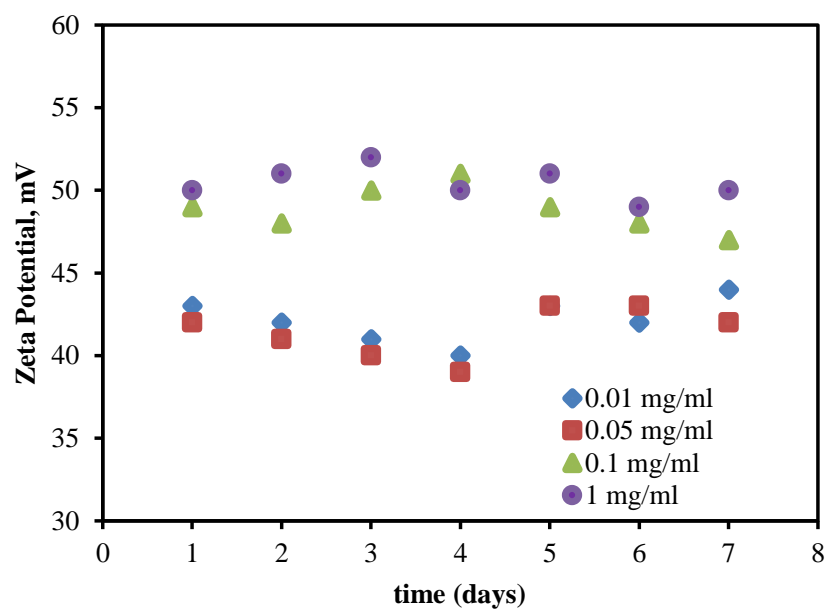

Figure 7. Stability of FA-NGO in aqueous solutions

\section{Conclusions}

In this study, synthesis of graphene oxide nanoparticles was performed by employing the improved Hummers' method, and it was activated by the folic acid antibody to increase the targeting ability after attachment of the drug to 
the nanostructure systems. SEM, FTIR, XRD, UV/Vis spectroscopy, and zeta potential analysis were performed for characterization of the products. The highest absorbance of the FA-NGO/DIW nanostructures produced at the concentration of $0.01 \mathrm{mg} / \mathrm{ml}-0.05 \mathrm{mg} / \mathrm{ml}$ synthesized by the Hummers' method and in the UV/Vis spectra, peaks at 232 $\mathrm{nm}$ and $270 \mathrm{~nm}$ corresponds to NGO-DIW and FANGO/DIW, respectively. The NGO-DIW and NGO-FADIW samples maintained stability for days. There is a direct correlation between zeta potential and the stability period of nanomaterials in aqueous solutions, i.e., with an increase in the absolute value of zeta potential, the stability period increases. Consequently, it is determined that the stability of the prepared samples is preserved for days. It is possible to develop a new drug delivery system which is based on FAconjugated NGO for the loading and targeted delivery of anticancer drugs in future studies. Furthermore, loading in a controlled manner and targeted delivery of mixed anticancer drugs by utilizing the graphene-based nanocarriers in question can be implemented in biomedicine in a widespread way.

\section{Acknowledgment}

This study is supported by TÜBİTAK BİDEB Authority under the project numbered 2209/A program 1919B011602924. We would like to thank TUBITAK Scientific Human Resource Support Program for their support in conducting this research..

\section{Nomenclature}

$\begin{array}{ll}N G O & : \text { Graphene oxide nanoparticle } \\ F A & : \text { Folic acid } \\ \text { DIW } & : \text { Deionized water }\end{array}$

\section{References}

1. Warheit, D. B., Sayes, C. M., Reed, K. L., \& Swain, K. A. Health effects related to nanoparticle exposures: environmental, health and safety considerations for assessing hazards and risks. Pharmacology \& therapeutics, 2008, 120 (1), 35-42.

2. Gonzalez, L., Lison, D., \& Kirsch-Volders, M. Genotoxicity of engineered nanomaterials: a critical review. Nanotoxicology, 2008, 2(4), 252-273.

3. Geim, Andre K., and Konstantin S. Novoselov. The rise of graphene, Nature materials, 2007, 6.3, 183.

4. Neto, A. C., Guinea, F., Peres, N. M., Novoselov, K. S., \& Geim, A. K. The electronic properties of graphene. Reviews of modern physics, 2009, 81(1), 109.

5. Becerril, H. A., Mao, J., Liu, Z., Stoltenberg, R. M., Bao, Z., \& Chen, Y. Evaluation of solution-processed reduced graphene oxide films as transparent conductors. ACS nano, 2008, 2(3), 463-470.

6. Stankovich, S., Dikin, D. A., Dommett, G. H., Kohlhaas, K. M., Zimney, E. J., Stach, E. A., ... \& Ruoff, R. S. Graphene-based composite materials. nature, 2006, 442(7100), 282.

7. Wang, C., Li, D., Too, C. O., \& Wallace, G. G. Electrochemical properties of graphene paper electrodes used in lithium batteries. Chemistry of Materials, 2009, 21(13), 2604-2606.
8. Pasricha, R., Gupta, S., \& Srivastava, A. K. A Facile and Novel Synthesis of Ag-Graphene-Based Nanocomposites. Small, 2009, 5(20), 2253-2259.

9. Shi, Y., Fang, W., Zhang, K., Zhang, W., \& Li, L. J. Photoelectrical Response in Single-Layer Graphene Transistors. Small, 2009, 5(17), 2005-2011.

10. Lv, X., Huang, Y., Liu, Z., Tian, J., Wang, Y., Ma, Y., ... $\&$ Chen, Y. Photoconductivity of Bulk-Film-Based Graphene Sheets. Small, 2009, 5(14), 1682-1687.

11. Dhand, V., Rhee, K. Y., Kim, H. J., \& Jung, D. H. A comprehensive review of graphene nanocomposites: research status and trends. Journal of Nanomaterials, 2013, 158.

12. Marcano, D. C., Kosynkin, D. V., Berlin, J. M., Sinitskii, A., Sun, Z., Slesarev, A., ... \& Tour, J. M. Improved synthesis of graphene oxide. ACS nano, 2010, 4(8), 48064814.

13. Sun, Z., Yan, Z., Yao, J., Beitler, E., Zhu, Y., \& Tour, J. M. Growth of graphene from solid carbon sources. Nature, 2010, 468(7323), 549.

14. Urbas, K., Aleksandrzak, M., Jedrzejczak, M., Jedrzejczak, M., Rakoczy, R., Chen, X., \& Mijowska, E. Chemical and magnetic functionalization of graphene oxide as a route to enhance its biocompatibility. Nanoscale research letters, 2014, 9(1), 656.

15. Yang, K., Feng, L., Hong, H., Cai, W., \& Liu, Z. Preparation and functionalization of graphene nanocomposites for biomedical applications. Nature protocols, 2013, 8(12), 2392.

16. Zhang, Y., Sun, C., Kohler, N., \& Zhang, M. Selfassembled coatings on individual monodisperse magnetite nanoparticles for efficient intracellular uptake. Biomedical microdevices, 2004, 6(1), 33-40.

17. Deb, A., \& Vimala, R. Camptothecin loaded graphene oxide nanoparticle functionalized with polyethylene glycol and folic acid for anticancer drug delivery. Journal of Drug Delivery Science and Technology, 2018, 43, 333-342.

18. Huang, Peng, et al. Folic acid-conjugated graphene oxide loaded with photosensitizers for targeting photodynamic therapy. Theranostics, 2011, 1: 240.

19. Low, Philip S.; Henne, Walter A.; Doorneweerd, Derek D. Discovery and development of folic-acid-based receptor targeting for imaging and therapy of cancer and inflammatory diseases. Accounts of chemical research, 2007, 41.1: 120-129.

20. Qin, X. C., Guo, Z. Y., Liu, Z. M., Zhang, W., Wan, M. M., \& Yang, B. W. Folic acid-conjugated graphene oxide for cancer targeted chemo-photothermal therapy. Journal of photochemistry and photobiology B: Biology, 2013, 120, 156-162.

21. Hummers Jr, W. S., \& Offeman, R. E. Preparation of graphitic oxide. Journal of the american chemical society, 1958, 80(6), 1339-1339.

22. Kovtyukhova, N. I., Ollivier, P. J., Martin, B. R., Mallouk, T. E., Chizhik, S. A., Buzaneva, E. V., \& Gorchinskiy, A. D. Layer-by-layer assembly of ultrathin composite films from micron-sized graphite oxide sheets and polycations. Chemistry of materials, 1999, 11(3), 771-778.

23. Zhang, L., Xia, J., Zhao, Q., Liu, L., \& Zhang, Z. Functional graphene oxide as a nanocarrier for controlled loading and targeted delivery of mixed anticancer drugs. Small, 2010, 6(4), 537-544.

24. Keklikcioğlu Çakmak, N, Temel, Ü, Yapıcı, K. Examination Of Rheological Behavior Of Water-Based Graphene Oxide Nanofluids. Cumhuriyet Science Journal, 2017, 38 (4), 176-183.

25. Szabó, T., Berkesi, O., \& Dékány, I. DRIFT study of deuterium-exchanged graphite oxide. Carbon, 2005, 43(15), 3186-3189. 
26. Pradhan, S. K., Xiao, B., Mishra, S., Killam, A., \& Pradhan, A. K. Resistive switching behavior of reduced graphene oxide memory cells for low power nonvolatile device application. Scientific reports, 2016, 6, 26763.

27. Angelopoulou, A., Voulgari, E., Diamanti, E. K., Gournis, D., \& Avgoustakis, K. Graphene oxide stabilized by PLAPEG copolymers for the controlled delivery of paclitaxel. European Journal of Pharmaceutics and Biopharmaceutics, 2015, 93, 18-26.

28. Reed, B. W., \& Sarikaya, M. Electronic properties of carbon nanotubes by transmission electron energy-loss spectroscopy. Physical Review B, 2001, 64(19), 195404. 